\title{
ON PRIMES OF DEGREE ONE IN FUNCTION FIELDS
}

\author{
GREG W. ANDERSON AND ROBERT INDIK
}

\begin{abstract}
We show that over the algebraic closure of a finite field, every point of the jacobian of a curve annihilated by a power of a prime $l$ is the $l$-primary component of a point in the image of the curve.
\end{abstract}

Let $X$ be a smooth, projective, geometrically connected curve of genus $g>0$ defined over the algebraic closure $\overline{\mathbf{F}}_{q}$ of the field $\mathbf{F}_{q}$ of $q$ elements. Fixing a basepoint $x_{0}$ of $X$ in $\overline{\mathbf{F}}_{q}$, let $\varphi: X \rightarrow J$ denote the embedding assigning to each point $x$ of $X$ the divisor class of the difference of $x$ and $x_{0}$. Let $l$ be any prime number and let $\lambda$ : $J\left(\overline{\mathbf{F}}_{q}\right) \rightarrow J\left(\overline{\mathbf{F}}_{q}\right)$, denote the projection of the torsion group $J\left(\overline{\mathbf{F}}_{q}\right)$ onto its l-primary component. The object of this note is to prove the following

TheOREM. The map $\lambda \circ \varphi: X\left(\overline{\mathbf{F}}_{q}\right) \rightarrow J\left(\overline{\mathbf{F}}_{q}\right)$, is surjective.

For the proof we need a lemma giving control over the distribution of primes of degree one in arithmetic progressions. Let $K / k$ be an abelian unramified extension of global fields of positive characteristic. Let $g$ be the genus of $k$ and suppose that $\mathbf{F}_{q}$ is the field of constants of both $K$ and $k$. For each prime $v$ of $k$ let $F_{v} \in \operatorname{Gal}(K / k)$ denote the corresponding arithmetic Frobenius.

LEMma. If there exists $\sigma_{0} \in \operatorname{Gal}(K / k)$ such that $F_{v} \neq \sigma_{0}$ for all primes $v$ of $k$ of degree one, then $q \leqslant(2 g[K: k]+3)^{2}$.

Proof. By hypothesis

$$
\sum_{l} 1=-\sum_{v} \sum_{\psi} \overline{\psi\left(\sigma_{0}\right)} \psi\left(F_{v}\right)
$$

where $v$ runs through all the primes of $k$ of degree one (i.e., of residue field coinciding with $\mathbf{F}_{q}$ ), and $\psi$ runs through all the nontrivial complex-valued characters of $\operatorname{Gal}(K / k)$. Now by the Riemann Hypothesis (see Appendix 5 of [W]) the left side of (*) is bounded below by $q+1-2 g \sqrt{q}$; the right side is bounded above by $([K: k]-1)(2 g-2) \sqrt{q}$. The desired conclusion follows immediately.

Turning now to the proof of the theorem, suppose that some point $d \in J\left(\overline{\mathbf{F}}_{q}\right)$ fails to be in the image of $\lambda \circ \varphi$. Assume, as is permissible, that $X$ is the base-change of a smooth projective curve $X_{0}$ defined over $\mathbf{F}_{q}, x_{0}$ is $\mathbf{F}_{q}$-rational, and $d$ is an $\mathbf{F}_{q}$-rational point of the jacobian $J_{0}$ of $X_{0}$. Fix a rational prime $r$ distinct from $l$. Let $k$ denote the function field of $X_{0}, \bar{k}$ an algebraic closure of $k$, and $v_{0}$ the prime of degree one of $k$

Received by the editors April 3, 1984

1980 Mathematics Subject Classification. Primary 12A80; Secondary 14H99.

1985 American Mathematical Society $0002-9939 / 85 \$ 1.00+\$ .25$ per page 
to which $x_{0}$ corresponds. For each positive integer $n$ let $k_{n}$ denote the compositum in $\bar{k}$ of $k$ and the unique extension of $\mathbf{F}_{q}$ in $\bar{k}$ of degree $r^{n}$. Let $K_{n}$ denote the maximal unramified abelian extension of $k_{n}$ in $\bar{k}$ of degree a power of $l$ in which $v_{0}$ splits completely. Letting $\alpha_{1}, \ldots, \alpha_{2 g}$ denote the reciprocal roots of the numerator of the zeta function of $X_{0}$ over $\mathbf{F}_{q}$, we have

$$
\left|\left[K_{n}: k_{n}\right]\right|_{l}=\prod_{j=1}^{2 g}\left|1-\alpha_{j}^{r^{n}}\right|_{l},
$$

where $\mid$ ? $\mid$, is any extension to $\overline{\mathbf{Q}}$ of the $l$-adic absolute value of $\mathbf{Q}$, whence $\left[K_{n}: k_{n}\right]$ is bounded. But for all $n$ the Artin symbol $\left(d, K_{n} / k_{n}\right) \in \operatorname{Gal}\left(K_{n} / k_{n}\right)$ fails to equal the arithmetic Frobenius $F_{v}$ for all primes $v$ of $k_{n}$ of degree one, whence, via the lemma, an estimate

$$
q^{r^{\prime \prime}} \leqslant\left(2 g\left[K_{n}: k_{n}\right]+3\right)^{2},
$$

a contradiction. This proves the theorem.

Acknowledgement. Thanks to Robert Coleman for suggesting the problem.

\section{REFERENCES}

[W ] A. Weil, Basic number theory, Springer-Verlag, New York, 1974.

Dipartment of Mathematics, HaRvard University, Cambridge, MassachusetTs 02138

Department of Mathematics, Brandeis University, Waltham, Massachusetts 02254 (Current address of Robert Indik)

Current address (G. W. Anderson): Department of Mathematics, University of California, Berkeley, California 94720 